\title{
Correction to "Inhibition of Glucose-6-Phosphate Dehydrogenase Activity Attenuates Right Ventricle Pressure and Hypertrophy Elicited by VEGFR Inhibitor + Hypoxia"
}

\begin{abstract}
In the above article [Kitagawa A, Jacob C, Jordan A, Waddell I, McMurtry IF, and Gupte SA (2021) J Pharmacol Exp Ther, 377:284-292; DOI: https://doi.org/10.1124/jpet.120.000166], a compositor error resulted in omitting the Nx and NX +4091 values in the first two columns for $\mathrm{RV} \mathrm{dP/dt} \max (\mathrm{mmHg} / \mathrm{s})$ in Table 1. The corrected Table 1 is provided below.

The PDF and HTML versions of the article have been corrected.

The compositor apologizes for any inconvenience caused by these errors.
\end{abstract}

TABLE 1

$\mathrm{RV}$ contractility and hypertrophy in $(\mathrm{Nx})$-control versus $\mathrm{PH}$ groups.The statistical differences between groups was determined by two-way ANOVA followed by Sidak's post hoc test.

\begin{tabular}{|c|c|c|c|c|c|c|}
\hline & $\begin{array}{c}\mathrm{Nx} \\
(n=11)\end{array}$ & $\begin{array}{c}\mathrm{Nx}+4091 \\
\quad(n=6)\end{array}$ & $\underset{(n=9)}{\mathrm{Hx}}$ & $\begin{array}{c}\mathrm{Hx}+4091 \\
(n=11)\end{array}$ & $\begin{array}{c}\mathrm{Hx}+\mathrm{SU} \\
(n=7)\end{array}$ & $\begin{array}{c}\mathrm{Hx}+\underset{(n=6)}{\mathrm{SU}+4091} \\
(n=6)\end{array}$ \\
\hline $\mathrm{nmHg} / \mathrm{s})$ & $1664 \pm 391$ & $1343 \pm 289$ & $2567 \pm 1745$ & $1437 \pm 390$ & $2706 \pm 703^{*}$ & $1647 \pm 114^{9}$ \\
\hline $\mathrm{n}(\mathrm{mmHg} / \mathrm{s})$ & $5266 \pm 1029$ & $5890 \pm 570$ & $4945 \pm 788$ & $4753 \pm 553$ & $4648 \pm 1099$ & $4504 \pm 1302$ \\
\hline
\end{tabular}

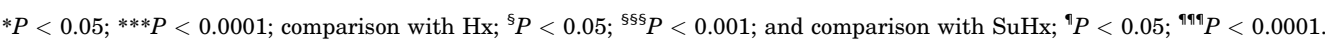

